Motion of Vortices in Type II Superconductors

Author(s): S. Jonathan Chapman and G. Richardson

Source: SIAM Journal on Applied Mathematics, Vol. 55, No. 5 (Oct., 1995), pp. 1275-1296

Published by: Society for Industrial and Applied Mathematics

Stable URL: http://www.jstor.org/stable/2102575

Accessed: 29/10/2009 16:12

Your use of the JSTOR archive indicates your acceptance of JSTOR's Terms and Conditions of Use, available at http://www.jstor.org/page/info/about/policies/terms.jsp. JSTOR's Terms and Conditions of Use provides, in part, that unless you have obtained prior permission, you may not download an entire issue of a journal or multiple copies of articles, and you may use content in the JSTOR archive only for your personal, non-commercial use.

Please contact the publisher regarding any further use of this work. Publisher contact information may be obtained at http://www.jstor.org/action/showPublisher?publisherCode=siam.

Each copy of any part of a JSTOR transmission must contain the same copyright notice that appears on the screen or printed page of such transmission.

JSTOR is a not-for-profit service that helps scholars, researchers, and students discover, use, and build upon a wide range of content in a trusted digital archive. We use information technology and tools to increase productivity and facilitate new forms of scholarship. For more information about JSTOR, please contact support@jstor.org. 


\title{
MOTION OF VORTICES IN TYPE II SUPERCONDUCTORS*
}

\author{
S. JONATHAN CHAPMAN ${ }^{\dagger}$ AND G. RICHARDSON ${ }^{\dagger}$
}

\begin{abstract}
The methods of formal asymptotics are used to examine the behaviour of a system of curvilinear vortices in a type II superconductor as the thickness of the vortex cores tends to zero. The vortices then appear as singularities in the field equation and are analagous to line vortices in inviscid hydrodynamics. A local analysis near each vortex core gives an equation of motion governing the evolution of these singularities.
\end{abstract}

Key words. superconductivity, vortices

AMS subject classifications. 82D55

1. Introduction. When a superconducting material is placed in an applied magnetic field of strength $H_{0}$, the state it adopts is conveniently described by Fig. 1. Here $\kappa$ is a material parameter which determines the type of superconducting material; $\kappa<1 / \sqrt{2}$ describes what is known as a type I superconductor, and $\kappa>1 / \sqrt{2}$ describes what is known as a type II superconductor.

For type I superconductors, if the magnetic field strength is sufficiently low, then the field will be excluded from the interior of the material. (This is known as the Meissner effect.) If, however, the field strength exceeds the critical value $H_{c}$, then it can no longer be excluded and the sample reverts to the normally conducting (normal) state, with the field penetrating it fully.

Type II superconductors are characterised by the existence of a third state, known as the "mixed state," in which there is a partial penetration of the magnetic field into the superconducting material. The mixed state consists of many normal filaments embedded in a superconducting matrix. Each filament carries with it a quantised amount of magnetic flux and is circled by a supercurrent vortex; thus these filaments are often known as vortices (see Fig. 2).

It is important to understand the behaviour of the superconductor while it is in the mixed state, since it is in this state that most superconductors appear in applications. The motion of vortices is of particular interest, since this motion dissipates energy and generates an electric field, which in turn results in an effective resistivity for the material.

The aim of this paper is to apply the methods of formal asymptotics to a system of curvilinear vortices in an unbounded superconductor in three dimensions for the case of an extreme type II material (i.e., as the parameter $\kappa \rightarrow \infty$ ). The vortices will behave as singularities in the field equation, rather like line vortices in inviscid hydrodynamics. A local examination of each vortex core will then give a "law of motion" for the evolution of these singularities.

A similar analysis has been applied recently to two-dimensional (i.e., rectilinear, parallel) vortices by Peres and Rubinstein [12] and Dorsey [8]. We aim to generalise their results to three-dimensional curvilinear vortices. We note also the work of Gor'kov and Kopnin [11], which has some parallels with the present work.

* Received by the editors February 25, 1994; accepted for publication (in revised form) September 6,1994 .

† Mathematical Institute, University of Oxford, 24-29 St. Giles', Oxford OX1 3LB, United Kingdom. The research of the first author was supported by a British Nuclear Electric Research fellowship. The research of the second author was supported by a Science and Engineering Research Council studentship. 


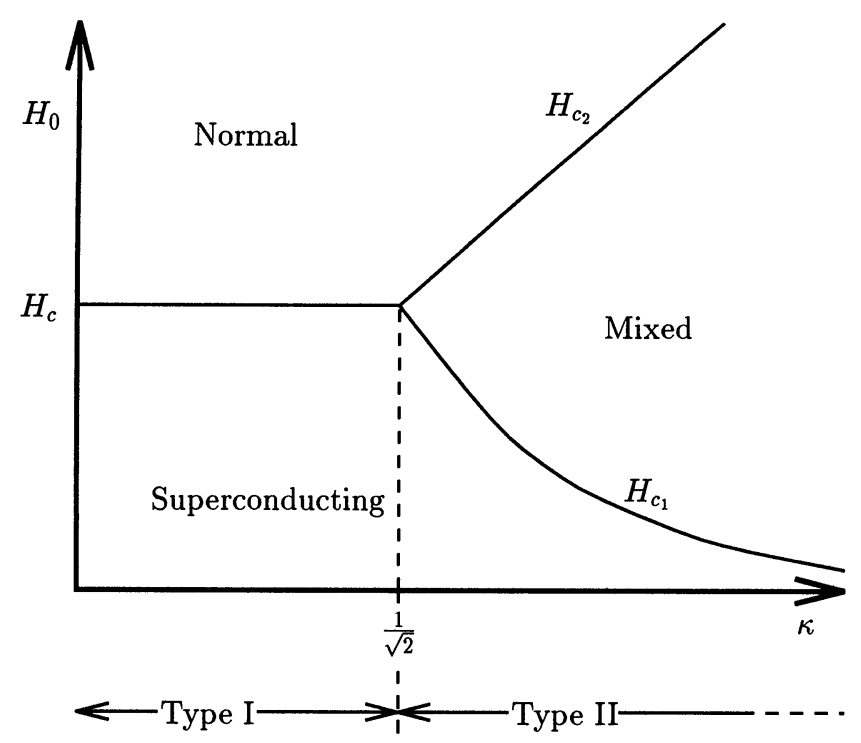

FIG. 1. The response of a superconducting material as a function of the applied magnetic field $H_{0}$ and the Ginzburg-Landau parameter $\kappa$.

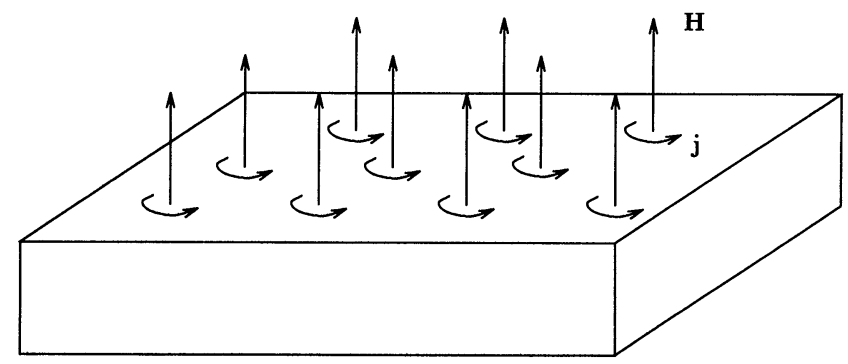

FIG. 2. Schematic diagram of the mixed state of a type II superconductor.

The model we work with is the time-dependent Ginzburg-Landau theory proposed by Gor'kov and Eliashberg [10]. In $\S 2$ we briefly present this theory and discuss the steady two-dimensional vortex solution. In $\S 3$ we perform a formal asymptotic analysis of a system of curvilinear vortices which are well separated (which will be made more precise later) and derive the law of motion governing the evolution of the system. In $\S 4$ we consider a system of densely packed vortices. Finally, in $\S 5$, we present our conclusions.

2. The Ginzburg-Landau theory. For a complete introduction to the Ginzburg-Landau theory of superconductivity the reader is referred to [4] and [7] and the references therein. Here we merely state the dimensionless, time-dependent Ginzburg-Landau equations proposed in [10] as

$$
\frac{\partial \Psi}{\partial t}+i \kappa \Psi \Phi+\kappa^{2}\left(\Psi|\Psi|^{2}-\Psi\right)-(\nabla-i \kappa \mathbf{A})^{2} \Psi=0
$$

$$
-\nabla \wedge(\nabla \wedge \mathbf{A})=\sigma\left(\frac{\partial \mathbf{A}}{\partial t}+\nabla \Phi\right)+\frac{i}{2 \kappa}\left(\Psi^{*} \nabla \Psi-\Psi \nabla \Psi^{*}\right)+|\Psi|^{2} \mathbf{A}
$$


where $\Psi$ is the complex superconducting order parameter (with $|\Psi|=1$ representing wholly superconducting material and $\Psi=0$ representing wholly normal material), $\Psi^{*}$ denotes the complex conjugate of $\Psi$, and $\mathbf{A}$ and $\Phi$ are the real magnetic vector potential, and electric scalar potential, respectively, which are such that

$$
\begin{aligned}
& \mathbf{H}=\nabla \wedge \mathbf{A} \\
& \mathbf{E}=-\frac{\partial \mathbf{A}}{\partial t}-\nabla \Phi,
\end{aligned}
$$

where $\mathbf{H}$ is the magnetic field and $\mathbf{E}$ is the electric field; $\mathbf{A}$ is unique up to the addition of a gradient; once $\mathbf{A}$ is given, $\Phi$ is unique up to the addition of a function of $t$. Here $\kappa$ and $\sigma$ are material constants; $\kappa$ is known as the Ginzburg-Landau parameter, while $\sigma$ is the dimensionless conductivity of the normal electrons in the material.

Lengths in (1)-(2) have been nondimensionalized with the penetration depth, which is a typical length associated with the variation of the magnetic field in the material. Time has been nondimensionalized with the timescale associated with the relaxation of $\Psi$ (so that the coefficient of $\partial \Psi / \partial t$ in (1) is unity).

In the steady state, with $\Phi=0$, equations (1)-(2) result from minimising the Ginzburg-Landau formulation of the Gibbs free energy [9]; in the time-dependent case they can be obtained as a limiting form of the microscopic BCS equations [1], [10] for certain materials. Note that, even though $\Psi$ is complex, (2) is in fact real, so we have five equations in six unknowns. This degeneracy is due to the property of gauge invariance, which we shall return to in a moment. A sixth equation can be provided by constraining the choice of gauge for $\mathbf{A}$, for example by requiring that $\operatorname{div} \mathbf{A}=0$.

Equations (1)-(2) exhibit vortex solutions, which can be illustrated by looking for a solution of the form

$$
\begin{aligned}
& \Psi=f(r) e^{i N \theta} \\
& \mathbf{A}=A(r) \mathbf{e}_{\theta},
\end{aligned}
$$

where $N$ is an integer, $r$ and $\theta$ are cylindrical polar coordinates, and $\mathbf{e}_{\theta}$ is the unit vector in the azimuthal direction. We find that $f$ and $A$ are solutions of

$$
\begin{aligned}
\frac{1}{\kappa^{2} r} \frac{d}{d r}\left(r \frac{d f}{d r}\right)-\left(A-\frac{N}{\kappa r}\right)^{2} f & =f^{3}-f, \\
\frac{d}{d r}\left(\frac{1}{r} \frac{d}{d r}(r A)\right) & =f^{2}\left(A-\frac{N}{\kappa r}\right), \\
f, \quad A \quad \text { bounded } \quad \text { as } r \rightarrow 0, & \text { as } r \rightarrow \infty .
\end{aligned}
$$

Berger and Chen [3] have shown the existence of a solution for $f$ and $A$ for all $N$ which necessarily has $f(0)=0$. The supercurrent is given by

$$
\mathbf{j}=-f^{2}\left(A-\frac{N}{r \kappa}\right) \mathbf{e}_{\theta}
$$


which demonstrates the vortex-like behaviour. The axial magnetic flux through the vortex is

$$
\int \mathbf{H} \cdot \mathbf{d} \mathbf{S}=\frac{2 \pi N}{\kappa}
$$

Thus the flux is quantised and a single "fluxon" has magnitude $2 \pi / \kappa$.

The Ginzburg-Landau parameter $\kappa$ is generally small for pure metals but quite large for superconducting alloys. Peres and Rubinstein give typical values of $\kappa=235$ for $\mathrm{Nb}_{3} \mathrm{Sn}, \kappa=640$ for $\mathrm{V}_{3} \mathrm{Ga}$, and $\kappa=785$ for $\mathrm{Nb}-\mathrm{N}$. They estimate $\sigma$ to be of the order of $10^{-3}-10^{-4}$. We will therefore be interested in the limit $\kappa \rightarrow \infty, \sigma \rightarrow 0$, with $\kappa^{2} \sigma=\sigma_{0}$ fixed. Our results hold under more general conditions, but the scaling we consider is the canonical one. We note that $\sigma \rightarrow 0$ implies that the timescale for the relaxation of the magnetic field is much shorter than the timescale for vortex motion, so that the magnetic field is effectively quasistatic.

To give an indication of what to expect from more complicated vortex lines, we perform a brief asymptotic analysis of (7)-(10) as $\kappa \rightarrow \infty$.

Away from the origin we find that the leading-order outer solution, denoted by the subscript $o$, satisfies

$$
\begin{array}{r}
f_{o}^{2}=1-A_{o}^{2}, \\
\frac{d}{d r}\left(\frac{1}{r} \frac{d}{d r}\left(r A_{o}\right)\right)=A_{o}-A_{o}^{3}, \\
A_{o} \quad \text { bounded as } r \rightarrow 0, \\
A_{o} \rightarrow 0 \text { as } r \rightarrow \infty .
\end{array}
$$

Hence $A_{o}=0, f_{o}=1$. Thus we find that away from the vortex core $f$ is unity to leading order and $A$ and $H$ are of order $1 / \kappa$. This is in agreement with (12).

Near the origin we rescale lengths by setting $r=R / \kappa$ to obtain the leading-order inner behaviour, denoted by the subscript $i$, as

$$
\begin{aligned}
\frac{1}{R} \frac{d}{d R}\left(R \frac{d f_{i}}{d R}\right)-\left(A_{i}-\frac{N}{R}\right)^{2} f_{i} & =f_{i}^{3}-f_{i}, \\
\frac{d}{d R}\left(\frac{1}{R} \frac{d}{d R}\left(R A_{i}\right)\right) & =0, \\
f_{i}, \quad A_{i} \quad \text { bounded as } r & \rightarrow 0, \\
f_{i} \rightarrow 1, \quad A_{i} \rightarrow 0 \text { as } r & \rightarrow \infty .
\end{aligned}
$$

Hence $A_{i}=0$,

$$
\frac{1}{R} \frac{d}{d R}\left(R \frac{d f_{i}}{d R}\right)-\frac{N^{2}}{R^{2}} f_{i}=f_{i}^{3}-f_{i}
$$

$f_{i}, \quad$ bounded as $r \rightarrow 0$,

$$
f_{i} \rightarrow 1 \text { as } r \rightarrow \infty \text {. }
$$


Not surprisingly, we will find that the leading-order behaviour near the vortex core of more general vortex lines will also be given by (21)-(23). The existence of a unique solution to (21)-(23) has been proved recently by Chen, Elliott, and Tang [6].

The fact that $A$ and $H$ are of order $1 / \kappa$ away from the vortex core motivates the following rescaling of the equations:

$$
\mathbf{A}=\frac{\mathbf{A}^{\prime}}{\kappa}, \quad \mathbf{H}=\frac{\mathbf{H}^{\prime}}{\kappa}, \quad \Phi=\frac{\Phi^{\prime}}{\kappa} .
$$

Dropping the primes, we now have

$$
\begin{gathered}
\frac{\partial \Psi}{\partial t}+i \Psi \Phi+\frac{1}{\epsilon^{2}}\left(\Psi|\Psi|^{2}-\Psi\right)-(\nabla-i \mathbf{A})^{2} \Psi=0 \\
-\nabla \wedge(\nabla \wedge \mathbf{A})=\sigma_{0} \epsilon^{2}\left(\frac{\partial \mathbf{A}}{\partial t}+\nabla \Phi\right)+\frac{i}{2}\left(\Psi^{*} \nabla \Psi-\Psi \nabla \Psi^{*}\right)+|\Psi|^{2} \mathbf{A},
\end{gathered}
$$

where $\epsilon=1 / \kappa$. As we mentioned earlier, these equations are gauge invariant in the sense that they are invariant under the transformations of the form

$$
\mathbf{A} \rightarrow \mathbf{A}+\nabla \omega, \quad \Phi \rightarrow \Phi-\frac{\partial \omega}{\partial t}, \quad \Psi \rightarrow \Psi e^{i \omega}
$$

We may write the eqations in terms of real variables by introducing the new, gaugeinvariant potentials

$$
\begin{gathered}
\mathbf{Q}=\mathbf{A}-\nabla \chi \\
\Theta=\Phi+\frac{\partial \chi}{\partial t},
\end{gathered}
$$

where $\Psi=f e^{i \chi}$ with $f$ and $\chi$ real. We then obtain coupled equations for just $f, \mathbf{Q}$, and $\Theta$ :

$$
\begin{aligned}
-\frac{\partial f}{\partial t}+\nabla^{2} f & =\frac{1}{\epsilon^{2}}\left(f^{3}-f\right)+f|\mathbf{Q}|^{2}, \\
f^{2} \Theta+\nabla \cdot\left(f^{2} \mathbf{Q}\right) & =0 \\
-\nabla \wedge(\nabla \wedge \mathbf{Q}) & =f^{2} \mathbf{Q}+\sigma_{0} \epsilon^{2}\left(\frac{\partial \mathbf{Q}}{\partial t}+\nabla \Theta\right),
\end{aligned}
$$

with

$$
\mathbf{H}=\nabla \wedge \mathbf{Q}
$$

Taking the divergence of (32) gives

$$
\nabla \cdot\left(f^{2} \mathbf{Q}\right)+\sigma_{0} \epsilon^{2}\left(\frac{\partial}{\partial t}(\nabla \cdot \mathbf{Q})+\nabla^{2} \Theta\right)=0
$$

which will prove useful later. 
3. Asymptotic analysis of a system of well-separated vortices. We consider the behaviour of a system of curvilinear vortices as $\epsilon \rightarrow 0$. We assume the vortices to be separated by distances of order one in the present nondimensionalization. This means that the distance between vortices is comparable of the lengthscale associated with the decay of magnetic field away for an isolated vortex (i.e., of the order of the penetration depth).

Far field. We denote the outer solution away from the vortex cores by the subscript $o$. Equation (30) implies that $f_{o}=1+O\left(\epsilon^{2}\right)$. Equations (31)-(32) give the leading-order behaviour of $\mathbf{Q}, \mathbf{H}$, and $\Theta$ as

$$
\begin{aligned}
\nabla \wedge\left(\nabla \wedge \mathbf{Q}_{o}^{(0)}\right)+\mathbf{Q}_{o}^{(0)} & =\mathbf{0}, \\
\Theta_{o}^{(0)} & =-\nabla \cdot \mathbf{Q}_{o}^{(0)}, \\
\nabla \wedge\left(\nabla \wedge \mathbf{H}_{o}^{(0)}\right)+\mathbf{H}_{o}^{(0)} & =-\nabla^{2} \mathbf{H}_{o}^{(0)}+\mathbf{H}_{o}^{(0)}=\mathbf{0} .
\end{aligned}
$$

We note that (35) implies that

$$
\nabla \wedge \mathbf{H}_{o}^{(0)}=-\mathbf{Q}_{o}^{(0)}
$$

which will prove useful later. To solve (37) we need to know the behaviour of $\mathbf{H}$ as a vortex is approached, which we will obtain by matching this outer solution with an inner solution near each vortex core.

Inner region. We consider an inner region in the vicinity of one of the vortex cores. Let the vortex lie along the curve $\Gamma$, given by $\mathbf{x}=(x, y, z)=\mathbf{q}(s, t)$. We define a local coordinate system $(s, \rho, \theta, T)$ by

$$
\begin{aligned}
\mathbf{x} & =\mathbf{q}(s, t)+\rho \cos \theta \hat{\mathbf{n}}(s, t)+\rho \sin \theta \hat{\mathbf{b}}(s, t), \\
t & =T
\end{aligned}
$$

where $s$ is arclength, $\hat{\mathbf{n}}(s, t)$ the unit normal, and $\hat{\mathbf{b}}(s, t)$ the unit binormal to the curve $\Gamma$. We define the inner coordinates by introducing the stretched variable $R$, defined by $\rho=\epsilon R$. The coordinate system so defined is not orthogonal away from the line $\mathbf{q}(s, t)$ when the torsion of the vortex is nonzero. However, it is approximately orthogonal, and the following expressions for $\nabla g, \nabla \cdot \mathbf{B}, \nabla^{2} g, \nabla \wedge \mathbf{B}$ and $\partial / \partial t$ in the inner region can be obtained:

$$
\begin{aligned}
\nabla g= & \frac{\partial g}{\partial s} \mathbf{e}_{\mathbf{s}}+\frac{1}{\epsilon} \frac{\partial g}{\partial R} \mathbf{e}_{\mathbf{R}}+\frac{1}{\epsilon R} \frac{\partial g}{\partial \theta} \mathbf{e}_{\theta}+O(\epsilon) \\
\nabla \cdot \mathbf{B}= & \frac{1}{\epsilon R}\left(\frac{\partial\left(R B_{R}\right)}{\partial R}+\frac{\partial B_{\theta}}{\partial \theta}\right)-C\left(B_{R} \cos \theta-B_{\theta} \sin \theta\right) \\
& +\frac{\partial B_{s}}{\partial s}+\tau \frac{\partial B_{s}}{\partial \theta}+O(\epsilon) \\
\nabla^{2} g= & \frac{1}{\epsilon^{2} R}\left(\frac{\partial}{\partial R}\left(R \frac{\partial g}{\partial R}\right)+\frac{1}{R} \frac{\partial^{2} g}{\partial \theta^{2}}\right) \\
& +\frac{C}{\epsilon}\left(\frac{1}{R} \frac{\partial g}{\partial \theta} \sin \theta-\frac{\partial g}{\partial R} \cos \theta\right)+O(1)
\end{aligned}
$$




$$
\begin{aligned}
\nabla \wedge \mathbf{B}=\frac{1}{\epsilon} & {\left[\frac{1}{R}\left(\frac{\partial}{\partial R}\left(R B_{\theta}\right)-\frac{\partial B_{R}}{\partial \theta}\right) \mathbf{e}_{\mathbf{s}}+\frac{1}{R} \frac{\partial B_{s}}{\partial \theta} \mathbf{e}_{\mathbf{R}}-\frac{\partial B_{s}}{\partial R} \mathbf{e}_{\theta}\right] } \\
& +\left[\tau\left(\frac{\partial B_{R}}{\partial \theta}-\frac{\partial}{\partial R}\left(R B_{\theta}\right)\right)+C \cos \theta B_{s}+\frac{\partial B_{R}}{\partial s}\right] \mathbf{e}_{\theta} \\
& +\tau R \frac{\partial B_{s}}{\partial R} \mathbf{e}_{\mathbf{s}}+\left(C \sin \theta B_{s}-\frac{\partial B_{\theta}}{\partial s}\right) \mathbf{e}_{\mathbf{R}}+O(\epsilon), \\
\frac{\partial}{\partial t}= & -\frac{1}{\epsilon}\left(\left(\mathbf{v} \cdot \mathbf{e}_{\mathbf{R}}\right) \frac{\partial}{\partial R}+\left(\mathbf{v} \cdot \mathbf{e}_{\theta}\right) \frac{1}{R} \frac{\partial}{\partial \theta}\right)+O(1),
\end{aligned}
$$

where $C$ is the curvature and $\tau$ is the torsion of the vortex line.

In the inner variables equations (33) and (32) become

$$
\begin{aligned}
\epsilon H_{i, s}= & \frac{1}{R}\left(\frac{\partial}{\partial R}\left(R Q_{i, \theta}\right)-\frac{\partial Q_{i, R}}{\partial \theta}\right)+\epsilon \tau R \frac{\partial Q_{i, s}}{\partial R}+O\left(\epsilon^{2} \mathbf{Q}_{i}\right) \\
\epsilon H_{i, R}= & \frac{1}{R} \frac{\partial Q_{i, s}}{\partial \theta}+\epsilon C \sin \theta Q_{i, s}-\epsilon \frac{\partial Q_{i, \theta}}{\partial s}+O\left(\epsilon^{2} \mathbf{Q}_{i}\right), \\
\epsilon H_{i, \theta}= & -\frac{\partial Q_{i, s}}{\partial R}+\epsilon \tau\left(\frac{\partial Q_{i, R}}{\partial \theta}-\frac{\partial}{\partial R}\left(R Q_{i, \theta}\right)\right) \\
& +\epsilon C \cos \theta Q_{i, s}+\epsilon \frac{\partial Q_{i, R}}{\partial s}+O\left(\epsilon^{2} \mathbf{Q}_{i}\right) \\
-\epsilon f_{i}^{2} Q_{i, s}= & \frac{1}{R}\left(\frac{\partial}{\partial R}\left(R H_{i, \theta}\right)-\frac{\partial H_{i, R}}{\partial \theta}\right)+\epsilon \tau R \frac{\partial H_{i, s}}{\partial R}+O\left(\epsilon^{2} \boldsymbol{\Theta}_{i}\right), \\
-\epsilon f_{i}^{2} Q_{i, R}= & \frac{1}{R} \frac{\partial H_{i, s}}{\partial \theta}+\epsilon C \sin \theta H_{i, s}-\epsilon \frac{\partial H_{i, \theta}}{\partial s}+O\left(\epsilon^{2} \boldsymbol{\Theta}_{i}\right), \\
-\epsilon f_{i}^{2} Q_{i, \theta}= & -\frac{\partial H_{i, s}}{\partial R}+\epsilon \tau\left(\frac{\partial H_{i, R}}{\partial \theta}-\frac{\partial}{\partial R}\left(R H_{i, \theta}\right)\right) \\
& +\epsilon C \cos \theta H_{i, s}+\epsilon \frac{\partial H_{i, R}}{\partial s}+O\left(\epsilon^{2} \boldsymbol{\Theta}_{i}\right)
\end{aligned}
$$

while equations (30), (31), and (34) become

$$
\begin{aligned}
\epsilon\left(\mathbf{v} \cdot \mathbf{e}_{\mathbf{R}}\right) & \frac{\partial f_{i}}{\partial R}+\left(\mathbf{v} \cdot \mathbf{e}_{\theta}\right) \frac{\epsilon}{R} \frac{\partial f_{i}}{\partial \theta}+\frac{1}{R}\left(\frac{\partial}{\partial R}\left(R \frac{\partial f_{i}}{\partial R}\right)+\frac{1}{R} \frac{\partial^{2} f_{i}}{\partial \theta^{2}}\right) \\
+ & \epsilon C\left(\frac{1}{R} \frac{\partial f_{i}}{\partial \theta} \sin \theta-\frac{\partial f_{i}}{\partial R} \cos \theta\right)=f_{i}^{3}-f_{i}+\epsilon^{2} f_{i}\left|\mathbf{Q}_{i}\right|^{2}+O\left(\epsilon^{2}\right) \\
\epsilon f_{i}^{2} \Theta_{i} & +\frac{1}{R}\left(\frac{\partial}{\partial R}\left(R f_{i}^{2} Q_{i, R}\right)+\frac{\partial}{\partial \theta}\left(f_{i}^{2} Q_{i, \theta}\right)\right) \\
& +-\epsilon C f_{i}^{2}\left(Q_{i, R} \cos \theta-Q_{i, \theta} \sin \theta\right)=O\left(\epsilon^{2} \mathbf{Q}_{i}, \epsilon Q_{i, s}\right)
\end{aligned}
$$




$$
\begin{gathered}
\frac{1}{R}\left(\frac{\partial}{\partial R}\left(R f_{i}^{2} Q_{i, R}\right)+\frac{\partial}{\partial \theta}\left(f_{i}^{2} Q_{i, \theta}\right)\right)-\epsilon C f_{i}^{2}\left(Q_{i, R} \cos \theta-Q_{i, \theta} \sin \theta\right) \\
-\epsilon \sigma_{0}\left[\left(\mathbf{v} \cdot \mathbf{e}_{\mathbf{R}}\right) \frac{\partial}{\partial R}+\left(\mathbf{v} \cdot \mathbf{e}_{\theta}\right) \frac{1}{R} \frac{\partial}{\partial \theta}\right]\left[\frac{1}{R} \frac{\partial}{\partial R}\left(R Q_{i, R}\right)+\frac{1}{R} \frac{\partial Q_{i, \theta}}{\partial \theta}\right] \\
+\epsilon \sigma_{0}\left(\frac{1}{R} \frac{\partial}{\partial R}\left(R \frac{\partial \Theta_{i}}{\partial R}\right)+\frac{1}{R^{2}} \frac{\partial^{2} \Theta_{i}}{\partial \theta^{2}}\right)=O\left(\epsilon^{2} \Theta_{i}, \epsilon Q_{i, s}\right) .
\end{gathered}
$$

Motivated by the elementary solution (5)-(6), since the vortex line appears rectilinear at leading order in the inner variables, we impose the following boundary conditions at the origin:

$$
f \rightarrow 0, \quad \mathbf{Q} \rightarrow-\frac{N_{k}}{\epsilon R} \mathbf{e}_{\theta} \quad \text { as } R \rightarrow 0 .
$$

In light of (55) we make the following ansatz for the leading-order behaviour of the inner solution, which we denote by the subscript $i$ :

$$
\begin{aligned}
\mathbf{Q}_{i} & \sim \frac{1}{\epsilon} Q_{i, \theta}^{(0)}(R) \mathbf{e}_{\theta}+\cdots, \\
f_{i} & \sim f_{i}^{(0)}(R)+\cdots .
\end{aligned}
$$

Substituting (56), (57) into (46)-(54) we find that the leading-order behaviour is given by

$$
\begin{aligned}
\mathbf{Q}_{i}^{(0)} & =-\frac{N_{k}}{R} \mathbf{e}_{\theta}, \\
f_{i}^{(0)^{\prime \prime}}+\frac{f_{i}^{(0)^{\prime}}}{R}-\frac{N_{k}^{2}}{R^{2}} f_{i}^{(0)} & =f_{i}^{(0)^{3}}-f_{i}^{(0)}, \\
f_{i}^{(0)}(0) & =0 \\
f_{i}^{(0)}(\infty) & =1,
\end{aligned}
$$

where the final boundary condition comes from matching with the outer solution. Equations (59)-(61) are exactly (21)-(23), obtained in $\S 2$ as the leading-order inner behaviour of a stationary rectilinear vortex. As noted in $\S 2$, the existence of a unique solution to (59)-(61) has been proved by Chen, Elliott, and Tang [6].

Far field. Matching (58) with the leading-order outer solution implies

$$
\mathbf{Q}_{o}^{(0)} \sim-\frac{N_{k}}{\rho} \mathbf{e}_{\theta} \quad \text { as } \quad \rho \rightarrow 0 .
$$

Equations (44) and (38) then give the asymptotic behaviour of $\mathbf{H}_{o}^{(0)}$ as

$$
\mathbf{H}_{o}^{(0)} \sim-N_{k} \log \rho \mathbf{e}_{\mathbf{s}} \quad \text { as } \rho \rightarrow 0 .
$$

We can now solve the outer problem (35), (37) together with the asymptotic behaviour (62), (63) as each vortex is approached. We effect the solution by noting that (37) with the behaviour (63) can be written as the single equation

$$
\nabla^{2} \mathbf{H}_{o}^{(0)}-\mathbf{H}_{o}^{(0)}=-2 \pi \sum_{m} \int_{\Gamma_{m}} N_{m} \delta\left(x-x_{m}(s)\right) \delta\left(y-y_{m}(s)\right) \delta\left(z-z_{m}(s)\right) \mathbf{d} \mathbf{s}
$$


where $\mathbf{q}_{m}(s)=\left(x_{m}(s), y_{m}(s), z_{m}(s)\right)$ and $\delta$ is the Dirac $\delta$-function. Noting that the Green's function satisfying

$$
\nabla^{2} G-G=-\delta(x) \delta(y) \delta(z) \quad \text { in } \mathbb{R}^{3}
$$

is

$$
G=\frac{1}{4 \pi} \frac{e^{-r}}{r}
$$

where $r=|\mathbf{x}|$, we see that the solution of $(64)$ is

$$
\mathbf{H}_{o}^{(0)}=\frac{1}{2} \sum_{m} N_{m} \int_{\Gamma_{m}} \frac{e^{-Z_{m}}}{Z_{m}} \mathbf{d s},
$$

where $\mathbf{Z}_{\mathbf{m}}=\mathbf{x}-\mathbf{q}_{\mathbf{m}}, Z_{m}=\left|\mathbf{Z}_{\mathbf{m}}\right|$, and $\Gamma_{m}$ is the curve representing the position of the $m$ th vortex. Applying (38) to (67) we find $\mathbf{Q}_{o}^{(0)}$ is given by

$$
\mathbf{Q}_{o}^{(0)}=-\frac{1}{2} \sum_{m} N_{m} \int_{\Gamma_{m}} \nabla\left(\frac{e^{-Z_{m}}}{Z_{m}}\right) \wedge \mathbf{d s} .
$$

At this point it is useful to elaborate on the analogy between vortices in a superconductor and vortices in an inviscid, otherwise irrotational fluid. For a similar system of fluid vortices $\Gamma_{m}$, the fluid velocity $\mathbf{u}$ satisfies

$$
\begin{aligned}
\operatorname{div} \mathbf{u} & =0 \\
\operatorname{curl} \mathbf{u} & =2 \pi \sum_{m} \int_{\Gamma_{m}} K_{m} \delta\left(x-x_{m}(s)\right) \delta\left(y-y_{m}(s)\right) \delta\left(z-z_{m}(s)\right) \mathbf{d} \mathbf{s},
\end{aligned}
$$

where $K_{m}$ are the vortex strengths (here not required to be integer valued) and $\mathbf{q}_{m}$ are parametrisations of the curves $\Gamma_{m}$ as before. The fluid velocity $\mathbf{u}$ is analogous to the electric current $\mathbf{J}$, which is given by

$$
\mathbf{J}_{o}^{(0)}=\operatorname{curl} \mathbf{H}_{o}^{(0)}=-\mathbf{Q}_{o}^{(0)}
$$

at leading order in the outer solution. The variable analogous to $\mathbf{H}_{o}^{(0)}$ is the vector potential $\boldsymbol{\Omega}$, which is such that

$$
\begin{gathered}
\operatorname{curl} \boldsymbol{\Omega}=\mathbf{u}, \\
\operatorname{div} \boldsymbol{\Omega}=0 .
\end{gathered}
$$

We have

$$
\nabla^{2} \Omega=-2 \pi \sum_{m} \int_{\Gamma_{m}} K_{m} \delta\left(x-x_{m}(s)\right) \delta\left(y-y_{m}(s)\right) \delta\left(z-z_{m}(s)\right) \mathbf{d s} .
$$

Hence

$$
\begin{aligned}
& \boldsymbol{\Omega}=\frac{1}{2} \sum_{m} K_{m} \int_{\Gamma_{m}} \frac{\mathbf{d s}}{Z_{m}}, \\
& \mathbf{u}=\frac{1}{2} \sum_{m} K_{m} \int_{\Gamma_{m}} \nabla\left(\frac{1}{Z_{m}}\right) \wedge \mathbf{d s} .
\end{aligned}
$$

Equation (76) is the familiar Biot-Savart integral. 
Before returning to the inner expansion we consider the asymptotic behaviour of (68) as the vortex line $\Gamma_{k}$ is approached, so that the distance $\rho$ from $\mathbf{x}$ to the nearest point on the vortex, $\mathbf{q}_{k}(S)$, becomes of order $\epsilon$. This will give motivation for the form of the inner expansion. The analysis parallels that performed on the Biot-Savart integral (76) by Saffman [13]. We write $\mathbf{Q}_{o}^{(0)}$ in the following form:

$$
\mathbf{Q}_{o}^{(0)}=\frac{N_{k}}{2}\left[\int_{S-L}^{S+L}+\int_{S+L}^{\infty}+\int_{-\infty}^{S-L}\right] e^{-Z_{k}}\left(\frac{1}{Z_{k}^{2}}+\frac{1}{Z_{k}^{3}}\right) \mathbf{Z}_{\mathbf{k}} \wedge \mathbf{d s}+\mathbf{Q}_{o, e x t}^{(0)}
$$

where

$$
\mathbf{Q}_{o, e x t}^{(0)}=-\frac{1}{2} \sum_{m \neq k} N_{m} \int_{\Gamma_{m}} \nabla\left(\frac{e^{-Z_{m}}}{Z_{m}}\right) \wedge \mathbf{d s}
$$

is the field due to other vortices and $1 /|\log \epsilon|^{\frac{1}{2}} \ll L \ll 1$. The major contribution to $\mathbf{Q}_{o}^{(0)}$ comes from the first integral in (77). We set $s=S+\epsilon l$ and expand

$$
\begin{aligned}
& \mathbf{q}_{k}(S+\epsilon l)-\mathbf{q}_{k}(S)=\epsilon l \hat{\mathbf{t}}(S)+\frac{\epsilon^{2} l^{2}}{2} C \hat{\mathbf{n}}(S)+\cdots, \\
& \begin{array}{r}
\mathbf{Z}_{\mathbf{k}}(S+\epsilon l)=\left(\epsilon R \cos \theta-\frac{\epsilon^{2} l^{2} C}{2}\right) \hat{\mathbf{n}}(S)+\epsilon R \sin \theta \hat{\mathbf{b}}(S) \\
-\epsilon l \hat{\mathbf{t}}(S)+\cdots,
\end{array}
\end{aligned}
$$

for $l \ll 1 / \epsilon$. Then

$$
\begin{aligned}
& \int_{S-L}^{S+L} e^{-Z_{k}}\left(\frac{1}{Z_{k}^{2}}+\frac{1}{Z_{k}^{3}}\right) \mathbf{Z}_{\mathbf{k}} \wedge \mathbf{d} \mathbf{s}=-\mathbf{e}_{\theta} \frac{1}{\epsilon R} \int_{-\frac{L}{\epsilon R}}^{\frac{L}{\epsilon R}} \frac{1}{\left(1+u^{2}\right)^{\frac{3}{2}}} d u \\
& \quad-\hat{\mathbf{b}}(S) \int_{-\frac{L}{\epsilon R}}^{\frac{L}{\epsilon R}} \frac{C u^{2}}{2\left(1+u^{2}\right)^{\frac{3}{2}}} d u-\mathbf{e}_{\theta} \frac{3 C \cos \theta}{2} \int_{-\frac{L}{\epsilon R}}^{\frac{L}{\epsilon R}} \frac{u^{2}}{\left(1+u^{2}\right)^{\frac{5}{2}}} d u+\cdots
\end{aligned}
$$

where $u=l / R$. Evaluating (82) we find that $\mathbf{Q}_{o}^{(0)}$ has the following asymptotic behaviour as the vortex line is approached:

$$
\begin{aligned}
\mathbf{Q}_{o}^{(0)}= & -\frac{N_{k}}{\epsilon R} \mathbf{e}_{\theta}(S)+\frac{N_{k} C}{2} \log (\epsilon R) \hat{\mathbf{b}}(S)-\frac{N_{k} C}{2}(\log 2-1) \hat{\mathbf{b}}(S) \\
& -\frac{N_{k} C}{2} \cos \theta \mathbf{e}_{\theta}(S)+\mathbf{Q}_{o, \text { ext }}^{(0)} \\
& +\frac{N_{k}}{2} \lim _{L \rightarrow 0}\left(\int_{|s-S|>L} e^{-Z_{k}}\left(\frac{1}{Z_{k}^{2}}+\frac{1}{Z_{k}^{3}}\right) \mathbf{Z}_{\mathbf{k}} \wedge \mathbf{d s}-\frac{1}{2} \log L \hat{\mathbf{b}}(s)\right)+\cdots
\end{aligned}
$$

It can be shown that the artificially introduced $\log L$ singularity cancels with the singular part of the remaining integral so that the limit in (83) gives a term of order one. 
Inner region. It now becomes clear how to proceed with the inner expansion. We expand $f_{i}, \mathbf{Q}_{i}, \mathbf{H}_{i}, \Theta_{i}$, and $\mathbf{v}_{i}$ as follows:

$$
\begin{aligned}
f_{i} & =f_{i}^{(0)}(R)+\epsilon \log \epsilon f_{i}^{(1)}+\epsilon f_{i}^{(2)}+\cdots, \\
\mathbf{Q}_{i} & =-\frac{N_{k}}{\epsilon R} \mathbf{e}_{\theta}+\log \epsilon \mathbf{Q}_{i}^{(1)}+\mathbf{Q}_{i}^{(2)}+\cdots, \\
\mathbf{H}_{i} & =\log \epsilon \mathbf{H}_{i}^{(0)}+\cdots, \\
\Theta_{i} & =\frac{\log \epsilon}{\epsilon} \Theta_{i}^{(0)}+\frac{1}{\epsilon} \Theta_{i}^{(1)}+\cdots, \\
\mathbf{v}_{i} & =\mathbf{v}^{(0)} \log \epsilon+\mathbf{v}^{(1)}+\cdots .
\end{aligned}
$$

Equating coefficients of $\log \epsilon$ in (46)-(54) gives

$$
\begin{aligned}
& \frac{1}{R}\left(\frac{\partial}{\partial R}\left(R Q_{i, \theta}^{(1)}\right)-\frac{\partial Q_{i, R}^{(1)}}{\partial \theta}\right)=0, \\
& \frac{\partial Q_{i, s}^{(1)}}{\partial \theta}=\frac{\partial Q_{i, s}^{(1)}}{\partial R}=0 \\
& \frac{1}{R}\left(\frac{\partial}{\partial R}\left(R H_{i, \theta}^{(0)}\right)-\frac{\partial H_{i, R}^{(0)}}{\partial \theta}\right)=0 \\
& \frac{\partial H_{i, s}^{(0)}}{\partial \theta}=\frac{\partial H_{i, s}^{(0)}}{\partial R}=0 \\
& \left(\mathbf{v}^{(0)} \cdot \mathbf{e}_{\mathbf{R}}\right) f_{i}^{(0)^{\prime}}(R)+\frac{1}{R} \frac{\partial}{\partial R}\left(R \frac{\partial f_{i}^{(1)}}{\partial R}\right)+\frac{1}{R^{2}} \frac{\partial^{2} f_{i}^{(1)}}{\partial \theta^{2}} \\
& =3 f_{i}^{(0)^{2}} f_{i}^{(1)}-f_{i}^{(1)}-\frac{2 N_{k} f_{i}^{(0)}}{R} \mathbf{e}_{\theta} \cdot \mathbf{Q}_{i}^{(1)}+\frac{N_{k}^{2} f_{i}^{(1)}}{R^{2}}, \\
& f_{i}^{(0)^{2}} \Theta_{i}^{(0)}-\frac{1}{R} \frac{\partial}{\partial \theta}\left(\frac{2 N_{k} f_{i}^{(0)} f_{i}^{(1)}}{R}\right)+\frac{1}{R} \frac{\partial}{\partial R}\left(R f_{i}^{(0)^{2}} \mathbf{Q}_{i, R}^{(1)}\right) \\
& +\frac{1}{R} \frac{\partial}{\partial \theta}\left(f_{i}^{(0)^{2}} \mathbf{Q}_{i, \theta}^{(1)}\right)=0, \\
& -\frac{1}{R} \frac{\partial}{\partial \theta}\left(\frac{2 N_{k} f_{i}^{(0)} f_{i}^{(1)}}{R}\right)+\frac{1}{R} \frac{\partial}{\partial R}\left(R f_{i}^{(0)^{2}} \mathbf{Q}_{i, R}^{(1)}\right)+\frac{1}{R} \frac{\partial}{\partial \theta}\left(f_{i}^{(0)^{2}} \mathbf{Q}_{i, \theta}^{(1)}\right) \\
& +\sigma_{0}\left(\frac{1}{R} \frac{\partial}{\partial R}\left(R \frac{\partial \Theta_{i}^{(0)}}{\partial R}\right)+\frac{1}{R^{2}} \frac{\partial^{2} \Theta_{i}^{(0)}}{\partial \theta^{2}}\right)=0 .
\end{aligned}
$$


From (91) and (92) and the fact that $\mathbf{H}$ must be divergence-free we find

$$
\mathbf{H}_{i}^{(0)}=\nabla w, \quad \nabla^{2} w=0 .
$$

Matching with the outer solution (63) gives

$$
\mathbf{H}_{i}^{(0)}=-N_{k} \mathbf{e}_{\mathbf{s}} .
$$

From (94) and (95) we find

$$
f_{i}^{(0)^{2}} \Theta_{i}^{(0)}=\sigma_{0}\left(\frac{1}{R} \frac{\partial}{\partial R}\left(R \frac{\partial \Theta_{i}^{(0)}}{\partial R}\right)+\frac{1}{R^{2}} \frac{\partial^{2} \Theta_{i}^{(0)}}{\partial \theta^{2}}\right) .
$$

It is apparent that $\Theta_{i}^{(0)}$ must possess a singularity on the vortex line due to the singularity of the phase parameter $\chi$ on this line. From (29) we have

$$
\Theta_{i}^{(0)} \sim-\frac{N_{k}}{R}\left(\mathbf{v}^{(0)} \cdot \mathbf{e}_{\theta}\right) \quad \text { as } R \rightarrow 0 .
$$

From (89) we see that we may write the $\mathbf{e}_{\theta}$ and $\mathbf{e}_{\mathbf{R}}$ components of $\mathbf{Q}_{i}^{(1)}$ in terms of a scalar potential $\psi_{1}$ :

$$
\begin{gathered}
\mathbf{Q}_{i, \theta}^{(1)}=\frac{1}{R} \frac{\partial \psi_{1}}{\partial \theta}, \\
\mathbf{Q}_{i, R}^{(1)}=\frac{\partial \psi_{1}}{\partial R} .
\end{gathered}
$$

In order to match with the asymptotic behaviour of the outer solution (83) we require that $\psi_{1} \sim$ constant $\times R \sin \theta$ as $R \rightarrow \infty$. With this motivation we look for a solution of the following form:

$$
\begin{aligned}
\psi_{1} & =\phi_{0}(R) \sin \theta, \\
\Theta_{i}^{(0)} & =U_{0} N_{k} \eta(R) \sin \theta, \\
f_{i}^{(1)} & =g_{0}(R) \cos \theta, \\
\mathbf{v}^{(0)} & =U_{0}\left(\cos \theta \mathbf{e}_{\mathbf{R}}-\sin \theta \mathbf{e}_{\theta}\right)=U_{0} \hat{\mathbf{n}} .
\end{aligned}
$$

The system of equations (93), (95), and (97) then reduces to

$$
\frac{1}{R}\left(R g_{0}\right)^{\prime}-3 f_{i}^{(0)}{ }^{2} g_{0}+g_{0}=-U_{0} f_{i}^{(0)^{\prime}}-\frac{2 N_{k} f_{i}^{(0)} \phi_{0}}{R^{2}}+\frac{g_{0}\left(N_{k}^{2}+1\right)}{R^{2}}
$$

$$
\begin{aligned}
\frac{1}{R}\left(R f_{i}^{(0)^{2}} \phi_{0}^{\prime}\right)^{\prime}+\sigma_{0} U_{0} N_{k}\left(\eta^{\prime}+\frac{\eta}{R}\right)^{\prime}-\frac{f_{i}^{(0)^{2}} \phi_{0}}{R^{2}}+\frac{2 N_{k} f_{i}^{(0)} g_{0}}{R^{2}} & =0 \\
\eta^{\prime \prime}+\frac{1}{R} \eta^{\prime}-\frac{1}{R^{2}} \eta-\frac{f_{i}^{(0)^{2}} \eta}{\sigma_{0}} & =0
\end{aligned}
$$

where $^{\prime} \equiv d / d R$. Equation (98) gives the boundary condition

$$
\eta \sim \frac{1}{R} \quad \text { as } R \rightarrow 0
$$


on equation (107). Matching with $\Theta_{o}^{(0)}$ gives the second condition:

$$
R \eta \rightarrow 0 \quad \text { as } R \rightarrow \infty .
$$

This gives a well-posed problem for $\eta$, and numerical solutions of (107) with boundary conditions (108) and (109) have been given by Peres and Rubinstein [12].

We consider (105) and (106). Noting that the derivative of the leading order solution $f_{i}^{(0)}$ satisfies the homogeneous version of equation (105) we see that there will be a solution if and only if a certain solvability condition is satisfied. To derive this condition we multiply (105) by $R f_{i}^{(0)^{\prime}}$ and the derivative of (59) by $R f_{i}^{(1)}$ and subtract to obtain

$$
R U_{0} f_{i}^{(0)^{\prime 2}}+\left(R g_{0} f_{i}^{(0)^{\prime}}-R g_{0} f_{i}^{(0)^{\prime \prime}}\right)^{\prime}=-\frac{2 N_{k} f_{i}^{(0)^{\prime}} f_{i}^{(0)} \phi_{0}}{R}+\frac{2 N_{k}^{2} f_{i}^{(0)} g_{0}}{R^{2}}
$$

Using (106) to eliminate the final $g_{0}$, integrating over $(0, \infty)$, and using the asymptotic behaviour of the $f_{i}^{(0)}$ and $\Theta_{i}^{(0)}$ at 0 and $\infty$ we find

$$
\lim _{R \rightarrow \infty}\left(\phi_{0}^{\prime}+\frac{\phi_{0}}{R}\right)=-\frac{U_{0} \beta}{N_{k}},
$$

where

$$
\beta=\int_{0}^{\infty} R f_{i}^{(0)^{\prime 2}} d R+N_{k}^{2} \int_{0}^{\infty} f_{i}^{(0)^{2}} \eta d R
$$

Hence

$$
\lim _{R \rightarrow \infty} \mathbf{Q}_{i}^{(1)}=-\frac{U_{0} \beta}{2 N_{k}} \hat{\mathbf{b}} .
$$

Before matching with the outer solution we proceed to determine one more term in the inner expansion. Equating coefficients at the next order in (46)-(54) gives

$$
\begin{gathered}
\frac{1}{R}\left(\frac{\partial}{\partial R}\left(R Q_{i, \theta}^{(2)}\right)-\frac{\partial Q_{i, R}^{(2)}}{\partial \theta}\right)=0 \\
\frac{\partial Q_{i, s}^{(2)}}{\partial \theta}=\frac{\partial Q_{i, s}^{(2)}}{\partial R}=0 \\
\left(\mathbf{v}^{(1)} \cdot \mathbf{e}_{\mathbf{R}}\right) f_{i}^{(0)^{\prime}}+\frac{1}{R} \frac{\partial}{\partial R}\left(R \frac{\partial f_{i}^{(2)}}{\partial R}\right)+\frac{1}{R^{2}} \frac{\partial^{2} f_{i}^{(2)}}{\partial \theta^{2}}-C f_{i}^{(0){ }^{\prime}} \cos \theta \\
=3 f_{i}^{(0)^{2}} f_{i}^{(2)}-f_{i}^{(2)}-2 \frac{N_{k} f_{i}^{(0)}}{R^{2}} \frac{\partial \psi_{2}}{\partial \theta}+\frac{N_{k}^{2} f_{i}^{(2)}}{R^{2}}, \\
f_{i}^{(0)^{2}} \Theta_{i}^{(1)}-\frac{2 N_{k} f_{i}^{(0)}}{R^{2}} \frac{\partial f_{i}^{(2)}}{\partial \theta}+\frac{1}{R} \frac{\partial}{\partial R}\left(R f_{i}^{(0)^{2}} \frac{\partial \psi_{2}}{\partial R}\right) \\
+\frac{f_{i}^{(0)^{2}}}{R^{2}} \frac{\partial^{2} \psi_{2}}{\partial \theta^{2}}+\frac{C N_{k} f_{i}^{(0)^{2}} \sin \theta}{R}=0,
\end{gathered}
$$




$$
\begin{aligned}
& -\frac{2 N_{k} f_{i}^{(0)}}{R^{2}} \frac{\partial f_{i}^{(2)}}{\partial \theta}+\frac{1}{R} \frac{\partial}{\partial R}\left(R f_{i}^{(0)^{2}} \frac{\partial \psi_{2}}{\partial R}\right)+\frac{f_{i}^{(0)^{2}}}{R^{2}} \frac{\partial^{2} \psi_{2}}{\partial \theta^{2}}-\frac{C N_{k} f_{i}^{(0)^{2}} \sin \theta}{R} \\
& +\sigma_{0}\left(\frac{1}{R} \frac{\partial}{\partial R}\left(R \frac{\partial \Theta_{i}^{(1)}}{\partial R}\right)+\frac{1}{R^{2}} \frac{\partial^{2} \Theta_{i}^{(1)}}{\partial \theta^{2}}\right)=0
\end{aligned}
$$

From (114) we see that again we may write the $\mathbf{e}_{\theta}$ and $\mathbf{e}_{\mathbf{R}}$ components of $\mathbf{Q}_{i}^{(2)}$ in terms of a scalar potential $\psi_{2}$ :

$$
\begin{aligned}
\mathbf{Q}_{i, \theta}^{(2)} & =\frac{1}{R} \frac{\partial \psi_{2}}{\partial \theta} \\
\mathbf{Q}_{i, R}^{(2)} & =\frac{\partial \psi_{2}}{\partial R} .
\end{aligned}
$$

Using (117) and (118) we see that $\Theta_{i}^{(1)}$ also satisfies (97). Matching with the outer solution gives $R \Theta_{i}^{(1)} \rightarrow 0$ as $R \rightarrow \infty$, while (29) gives $\Theta_{i}^{(1)} \sim-\frac{N_{k}}{R}\left(\mathbf{v}^{(1)} \cdot \mathbf{e}_{\theta}\right)$ as $R \rightarrow 0$. Motivated by this and the asymptotic behaviour of the outer solution (83), we seek a solution of (116)-(118) of the form

$$
\begin{aligned}
\psi_{2}= & \phi_{1}(R) \sin \theta+\phi_{2}(R) \sin (\theta-\alpha), \\
f_{i}^{(2)} & =g_{1}(R) \cos \theta+g_{2}(R) \cos (\theta-\alpha), \\
\Theta_{i}^{(1)} & =U_{1} N_{k} \eta(R) \sin \theta+U_{2} N_{k} \eta(R) \sin (\theta-\alpha), \\
\mathbf{v}^{(1)} & =U_{1} \hat{\mathbf{n}}+U_{2}(\cos \alpha \hat{\mathbf{n}}+\sin \alpha \hat{\mathbf{b}}) \\
& =U_{1}\left(\cos \theta \mathbf{e}_{\mathbf{R}}-\sin \theta \mathbf{e}_{\theta}\right)+U_{2}\left(\cos (\theta-\alpha) \mathbf{e}_{\mathbf{R}}-\sin (\theta-\alpha) \mathbf{e}_{\theta}\right) .
\end{aligned}
$$

Substituting (121)-(124) into (116)-(118) we obtain two sets of ordinary differential equations for $\left(\phi_{1}, g_{1}\right)$ and $\left(\phi_{2}, g_{2}\right)$, respectively. The equations for $\left(\phi_{2}, g_{2}\right)$ are identical to (105) and (106), with $g_{0}$ replaced by $g_{2}, \phi_{0}$ by $\phi_{2}$ and $U_{0}$ by $U_{2}$. Hence

$$
\phi_{2} \sim-\frac{U_{2} \beta R}{2 N_{k}} \quad \text { as } R \rightarrow \infty .
$$

The equations for $\left(\phi_{1}, g_{1}\right)$ are

$$
U_{1} f_{i}^{(0)^{\prime}}+\frac{1}{R}\left(R g_{1}^{\prime}\right)^{\prime}=3 f_{i}^{(0)^{2}} g_{1}-g_{1}-\frac{2 N_{k} f_{i}^{(0)} \phi_{1}}{R^{2}}+\frac{\left(N_{k}^{2}+1\right) g_{1}}{R^{2}}+C f_{i}^{(0)^{\prime}}
$$

(127) $\frac{1}{R}\left(R f_{i}^{(0)^{2}} \phi_{1}^{\prime}\right)^{\prime}+\sigma_{0} U_{1} N_{k}\left(\eta^{\prime}+\frac{\eta}{R}\right)^{\prime}-\frac{f_{i}^{(0)^{2}} \phi_{1}}{R^{2}}+\frac{2 N_{k} f_{i}^{(0)} g_{1}}{R^{2}}-\frac{C N_{k} f_{i}^{(0)^{2}}}{R}=0$.

A similar analysis to that leading to (116) can be employed. Multiplying (126) by $R f_{i}^{(0)^{\prime}}$, subtracting the derivative of (59) multiplied by $R g_{1}$, eliminating $g_{1}$ by (127), and integrating over $(0, R)$ gives

$$
\phi_{1} \sim \frac{N_{k} C R}{2}\left(\log R+\gamma-\frac{1}{2}-\frac{U_{1} \beta}{C N_{k}^{2}}\right) \quad \text { as } R \rightarrow \infty,
$$


where

$$
\gamma=\int_{0}^{1} \frac{f_{i}^{(0)^{2}}}{R} d R+\int_{1}^{\infty} \frac{f_{i}^{(0)^{2}}-1}{R} d R+\frac{1}{N_{k}^{2}} \int_{0}^{\infty} R f_{i}^{(0)^{2}} d R .
$$

Combining (125) and (128) we have

$$
\begin{aligned}
\mathbf{Q}_{i}^{(2)} \sim & -\frac{C N_{k}}{2}\left(\cos \theta \mathbf{e}_{\theta}-\left(\log R+\gamma+\frac{1}{2}-\frac{U_{1} \beta}{C N_{k}^{2}}\right) \hat{\mathbf{b}}\right) \\
& -\frac{U_{2} \beta}{2 N_{k}}(\cos \alpha \hat{\mathbf{b}}-\sin \alpha \hat{\mathbf{n}})+\mathbf{Q}_{i, s}^{(2)}(s) \mathbf{e}_{\mathbf{s}} \quad \text { as } R \rightarrow \infty
\end{aligned}
$$

Matching. We complete our analysis by matching the inner terms $\mathbf{Q}_{i}^{(1)}$ and $\mathbf{Q}_{i}^{(2)}$ with the outer solution $\mathbf{Q}_{o}^{(0)}$, using (113), (130), and (83), following the matching principle of Van Dyke [14]. We find that for the inner and outer solutions to match we must have

$$
\begin{aligned}
& \mathbf{v}^{(0)}=-\frac{C N_{k}^{2} \hat{\mathbf{n}}}{\beta} \\
& \mathbf{v}^{(1)}=\frac{C N_{k}^{2}}{\beta}\left(\gamma+\log 2-\frac{1}{2}\right) \hat{\mathbf{n}}-\frac{2 P N_{k}}{\beta}(\cos \alpha \hat{\mathbf{n}}+\sin \alpha \hat{\mathbf{b}})
\end{aligned}
$$

where

$$
\begin{aligned}
& P(\cos \alpha \hat{\mathbf{b}}-\sin \alpha \hat{\mathbf{n}})+Q_{o, s}^{(0)}(s) \hat{\mathbf{t}}(s)=\mathbf{Q}_{o, e x t}^{(0)} \\
& \quad+\frac{N_{k}}{2} \lim _{L \rightarrow 0}\left(\int_{|s-S|>L} e^{-Z_{k}}\left(\frac{1}{Z_{k}^{2}}+\frac{1}{Z_{k}^{3}}\right) \mathbf{Z}_{\mathbf{k}} \wedge \mathbf{d s}-\frac{1}{2} \log L \hat{\mathbf{b}}(s)\right)
\end{aligned}
$$

$\hat{\mathbf{t}}(s)$ is the unit vector tangent to the vortex line, and $\mathbf{Q}_{o, e x t}^{(0)}$ is given by (78). We note from equation (38) that

$$
\mathbf{J}_{o}^{(0)}=\operatorname{curl} \mathbf{H}_{o}^{(0)}=-\mathbf{Q}_{o}^{(0)}
$$

where $\mathbf{J}$ is the electric current. Writing

$$
\mathbf{J}_{o, e x t}^{(0)}=-\mathbf{Q}_{o, e x t}^{(0)}
$$

and

$$
\mathbf{J}_{o, f i n}^{(0)}=-\frac{N_{k}}{2} \lim _{L \rightarrow 0}\left(\int_{|s-S|>L} e^{-Z_{k}}\left(\frac{1}{Z_{k}^{2}}+\frac{1}{Z_{k}^{3}}\right) \mathbf{Z}_{\mathbf{k}} \wedge \mathbf{d s}-\frac{1}{2} \log L \hat{\mathbf{b}}(s)\right),
$$

we see that (132) may be written as

$$
\mathbf{v}^{(1)}=\frac{C N_{k}^{2}}{\beta}\left(\gamma+\log 2-\frac{1}{2}\right) \hat{\mathbf{n}}+\frac{2 N_{k}}{\beta}\left(\mathbf{J}_{o, e x t}^{(0)}+\mathbf{J}_{o, f i n}^{(0)}\right) \wedge \hat{\mathbf{t}} .
$$

Finally, combining (131) and (134), we obtain the following law for vortex motion:

$$
\mathbf{v}=-\frac{\log \epsilon C N_{k}^{2}}{\beta} \hat{\mathbf{n}}+\frac{C N_{k}^{2}}{\beta}\left(\gamma+\log 2-\frac{1}{2}\right) \hat{\mathbf{n}}+\frac{2 N_{k}}{\beta}\left(\mathbf{J}_{o, e x t}^{(0)}+\mathbf{J}_{o, f i n}^{(0)}\right) \wedge \hat{\mathbf{t}}
$$


The first two terms here correspond to the local self-induced motion of the vortex filament. The final term is often interpreted as the Lorentz force on a vortex line (since the vortex carries a quantised amount of magnetic flux).

Remark. If we had been considering a finite domain, rather than an infinite one, equation (135) would still be valid, provided that we interpret the meaning of $\mathbf{J}_{o \text { ext }}^{(0)}$ correctly. This term is the regular part of the solution of equation (64) evaluated at a particular vortex, having subtracted the contribution from the vortex itself. In the infinite case we were able to evaluate $\mathbf{J}_{o, \text { ext }}^{(0)}$ explicitly to give equation (78). In the finite case there will in general be no closed-form solution.

Calculation of the electric field. It is of interest to calculate the electric field associated with a moving vortex. In particular, this will allow us to determine the size of the voltage pulse generated by the vortex as a function of its velocity.

Outer region. The electric field is given by

$$
\mathbf{E}=-\frac{\partial \mathbf{Q}}{\partial t}-\nabla \Theta
$$

In the outer region, away from the vortex core, we expand

$$
\mathbf{E}_{o}=\mathbf{E}_{o}^{(0)}+\cdots
$$

The leading-order electric field is then given simply by

$$
\mathbf{E}_{o}^{(0)}=\frac{\partial}{\partial t}\left(\operatorname{curl} \mathbf{H}_{o}^{(0)}\right)
$$

which is the London equation.

Inner region. In the inner coordinates (136) becomes

$$
\mathbf{E}_{i}=\frac{1}{\epsilon}\left((\mathbf{v} \cdot \nabla) \mathbf{Q}_{i}-\nabla \Theta_{i}\right)+O(1) .
$$

We expand

$$
\mathbf{E}_{i}=\frac{\log \epsilon}{\epsilon^{2}} \mathbf{E}_{i}^{(0)}+\frac{1}{\epsilon^{2}} \mathbf{E}_{i}^{(1)}+\cdots
$$

Note that $\mathbf{E}_{i}$ is greater than $O\left(\epsilon^{-2}\right)$; hence the integral of $\mathbf{E}_{i}$ over the inner region is greater than $O(1)$, and the outer solution in fact sees a $\delta$-function electric field at the vortex core in the limit as $\epsilon \rightarrow 0$. This is the cause of the voltage pulse associated with moving vortices. We calculate the strength of this $\delta$-function by considering the limit as $\nu$ tends to zero and $X$ tends to infinity of

$$
\int_{\nu<R<X} \mathbf{E}_{i} \wedge \hat{\mathbf{t}} d S
$$

where $\hat{\mathbf{t}}$ is the tangent to the vortex line. Noting that

$$
\left(\mathbf{v}^{(0)} \cdot \nabla\right) \mathbf{Q}_{i}^{(0)}=-\mathbf{v}^{(0)} \wedge \operatorname{curl} \mathbf{Q}_{i}^{(0)}+\nabla\left(\mathbf{v}^{(0)} \cdot \mathbf{Q}_{i}^{(0)}\right)
$$


we find

$$
\begin{aligned}
& \mathbf{k} \cdot \int \mathbf{E}_{i}^{(0)} \wedge \hat{\mathbf{t}} d S \\
& =\int \mathbf{k} \cdot\left[-\left(\left(\mathbf{v}^{(0)} \wedge \mathbf{Q}_{i}^{(0)}\right) \wedge \hat{\mathbf{t}}\right)+\nabla\left(\mathbf{v}^{(0)} \cdot \mathbf{Q}_{i}^{(0)}-\Theta_{i}^{(0)}\right) \wedge \hat{\mathbf{t}}\right] d S \\
& =\int\left[-\left(\mathbf{k} \wedge\left(\mathbf{v}^{(0)} \wedge \operatorname{curl} \mathbf{Q}_{i}^{(0)}\right)\right) \cdot \hat{\mathbf{t}}+\mathbf{k} \wedge \nabla\left(\mathbf{v}^{(0)} \cdot \mathbf{Q}_{i}^{(0)}-\Theta_{i}^{(0)}\right) \cdot \hat{\mathbf{t}}\right] d S \\
& =\int\left[\left(\mathbf{k} \cdot \mathbf{v}^{(\mathbf{0})}\right) \operatorname{curl} \mathbf{Q}_{i}^{(0)} \cdot \hat{\mathbf{t}}-\operatorname{curl}\left(\left(\mathbf{v}^{(0)} \cdot \mathbf{Q}_{i}^{(0)}-\Theta_{i}^{(0)}\right) \mathbf{k}\right)\right] d S
\end{aligned}
$$

where $\mathbf{k}$ is an arbitrary vector in the plane normal to the vortex. Applying Stokes' theorem and using the fact that $\mathbf{k}$ was arbitrary, we find

$$
\begin{aligned}
\int \mathbf{E}_{i}^{(0)} \wedge \hat{\mathbf{t}} d S=\mathbf{v}^{(0)} \int_{R=X} \mathbf{Q}_{i}^{(0)} X \mathbf{e}_{\theta} d \theta-\mathbf{v}^{(0)} \int_{R=\nu} \mathbf{Q}_{i}^{(0)} \nu \mathbf{e}_{\theta} d \theta \\
\quad-\int_{R=X}\left(\mathbf{v}^{(0)} \cdot \mathbf{Q}_{i}^{(0)}-\Theta_{i}^{(0)}\right) X \mathbf{e}_{\theta} d \theta \\
\quad+\int_{R=\nu}\left(\mathbf{v}^{(0)} \cdot \mathbf{Q}_{i}^{(0)}-\Theta_{i}^{(0)}\right) \nu \mathbf{e}_{\theta} d \theta .
\end{aligned}
$$

When we insert the solution for $\mathbf{Q}_{i}^{(0)}$ and $\Theta_{i}^{(0)}$ into this expression we find

$$
\int \mathbf{E}_{i}^{(0)} \wedge \hat{\mathbf{t}} d S=N_{k} \pi \mathbf{v}^{(0)}
$$

Similarly, we find

$$
\int \mathbf{E}_{i}^{(1)} \wedge \hat{\mathbf{t}} d S=N_{k} \pi \mathbf{v}^{(1)}
$$

This result can be combined with the outer solution (137) to give the following expression for the electric field, valid up to $o(1)$ :

$$
\mathbf{E}_{o}=\frac{\partial}{\partial t}\left(\operatorname{curl} \mathbf{H}_{o}\right)+\boldsymbol{\delta} \wedge \mathbf{v}
$$

since

$$
\begin{aligned}
\lim _{\nu \rightarrow 0} \int_{r<\nu} \frac{\partial}{\partial t}\left(\operatorname{curl} \mathbf{H}_{o}\right) \wedge \hat{\mathbf{t}} d S & =-N_{k} \pi \mathbf{v} \\
\lim _{\nu \rightarrow 0} \int_{r<\nu}(\boldsymbol{\delta} \wedge \mathbf{v}) \wedge \hat{\mathbf{t}} d S & =2 N_{k} \pi \mathbf{v}
\end{aligned}
$$

where

$$
\boldsymbol{\delta}(x, y, z)=\int_{\Gamma} \delta\left(x-x^{\prime}\right) \delta\left(y-y^{\prime}\right) \delta\left(z-z^{\prime}\right) \mathbf{d} \mathbf{s}^{\prime} .
$$

4. Asymptotic analysis of a system of densely packed vortices. In the previous section we considered vortices that were separated by distances of order one. This generated an outer magnetic field that was also order one, and the leadingorder term in the equation of vortex motion was a local self-induced motion due to curvature. 
Here we consider briefly the case in which the vortex spacing is $O(\delta)$, where $\epsilon \ll \delta^{2} \ll 1$. This leads to an outer magnetic field which is of order $1 / \delta^{2}$. When we also choose $\delta^{2} \ll 1 /|\log \epsilon|$ we expect that the motion due to this outer field will replace the self-induced motion as the leading term in the law of vortex motion. We show that this is indeed the case. There will be three regions to consider, namely, $O(1), O(\delta)$, and $O(\epsilon)$ lengthscales.

Outer region. A complete determination of the outer expansions of $\mathbf{H}_{o}$ and $\mathbf{Q}_{o}$ will involve averaging over many vortices. Such a procedure is performed in [5], under the assumption that neighbouring vortices point in the same direction and move with the same velocity, and results in the equations

$$
\begin{aligned}
\mathbf{H}_{o} & =\frac{1}{\delta^{2}} \mathbf{H}_{o}^{(0)}+\cdots, \\
\mathbf{Q}_{o} & =\frac{1}{\delta^{2}} \mathbf{Q}_{o}^{(0)}+\cdots, \\
\omega_{t}+\operatorname{curl}\left(\omega \wedge \mathbf{v}^{(0)}\right) & =\mathbf{0}, \\
(\operatorname{curl})^{2} \mathbf{H}_{o}^{(0)}+\mathbf{H}_{o}^{(0)} & =\omega, \\
\mathbf{Q}_{o}^{(0)} & =-\operatorname{curl} \mathbf{H}_{o}^{(0)},
\end{aligned}
$$

where $\omega$ is the vortex density, $\mathbf{v}^{(0)}$ is the vortex velocity, and time has been rescaled with the inverse of the magnitude of this velocity, which we will find to be $\delta^{2}$. To close the model we need to determine $\mathbf{v}^{(0)}$, which will again be provided by our inner analysis of the vortex cores.

Intermediate region. When lengths are rescaled with $\delta$ we find that the leading-order equations for $\mathbf{H}$ and $\mathbf{Q}$ in the intermediate region, denoted by the subscript int, become

$$
\begin{aligned}
\operatorname{curl} \mathbf{H}_{\text {int }} & =\mathbf{0}, \\
\operatorname{curl} \mathbf{Q}_{\text {int }} & =\mathbf{0}, \\
\operatorname{div} \mathbf{H}_{\text {int }} & =0, \\
\operatorname{div} \mathbf{Q}_{\text {int }} & =0
\end{aligned}
$$

with the condition

$$
\mathbf{Q}_{\text {int }} \sim-\frac{N_{k}}{\delta r} \mathbf{e}_{\theta}
$$

as each vortex line is approached, where $r=\delta^{-1} R$ and $R$ and $\theta$ are the curvilinear coordinates (39)-(40) previously introduced. Hence

$$
\begin{aligned}
\mathbf{H}_{\mathrm{int}} & =\frac{\mathbf{H}_{\mathrm{int}}^{(0)}}{\delta^{2}}+\cdots, \\
\mathbf{Q}_{\mathrm{int}} & =\frac{\mathbf{Q}_{\mathrm{int}}^{(0)}}{\delta^{2}}-\frac{1}{2 \delta} \sum_{m} N_{m} \int_{\Gamma_{m}} \nabla\left(\frac{1}{Z_{m}}\right) \wedge \mathbf{d s}+\cdots, \\
f_{o} & =1+\cdots,
\end{aligned}
$$


where $\mathbf{H}_{\text {int }}^{(0)}$ and $\mathbf{Q}_{\text {int }}^{(0)}$ are constant and $Z_{m}$ and $\Gamma_{m}$ are as before; $\mathbf{H}_{\text {int }}^{(0)}$ and $\mathbf{Q}_{\text {int }}^{(0)}$ are therefore simply $\mathbf{H}_{o}^{(0)}$ and $\mathbf{Q}_{o}^{(0)}$ evaluated at the point about which lengths were rescaled.

Inner region. The inner expansion becomes

$$
\begin{aligned}
f & =f_{i}^{(0)}(R)+\frac{\epsilon}{\delta^{2}} f_{i}^{(1)}+\cdots \\
\mathbf{Q} & =-\frac{N_{k} \mathbf{e}_{\theta}}{\epsilon R}+\frac{1}{\delta^{2}} \mathbf{Q}_{i}^{(1)}+\log \epsilon \mathbf{Q}_{i}^{(2)}+\cdots, \\
\Theta & =\frac{1}{\epsilon \delta^{2}} \Theta_{i}^{(0)}+\frac{\log \epsilon}{\epsilon} \Theta_{i}^{(1)}+\cdots \\
\mathbf{H}_{i} & =\frac{1}{\delta^{2}} \mathbf{H}_{i}^{(0)}+\log \epsilon \mathbf{H}_{i}^{(1)}+\cdots \\
\mathbf{v} & =\frac{1}{\delta^{2}} \mathbf{v}^{(0)}+\log \epsilon \mathbf{v}^{(1)}+\cdots
\end{aligned}
$$

A scalar potential may be defined for $\mathbf{Q}_{i}^{(1)}$ as before:

$$
\begin{aligned}
\mathbf{Q}_{i, \theta}^{(1)} & =\frac{1}{R} \frac{\partial \psi_{1}}{\partial \theta}, \\
\mathbf{Q}_{i, R}^{(1)} & =\frac{\partial \psi_{1}}{\partial R} .
\end{aligned}
$$

The order- $\delta^{-2}$ solution of the inner equations then takes the form

$$
\begin{array}{r}
\psi_{1}=\phi_{0}(R) \sin (\theta-\alpha), \\
\Theta_{i}^{(0)}=U_{0} \eta(R) \sin (\theta-\alpha), \\
f_{i}^{(1)}=g_{0}(R) \cos (\theta-\alpha), \\
\mathbf{v}^{(0)}=U_{0}\left(\cos (\theta-\alpha) \mathbf{e}_{\mathbf{R}}-\sin (\theta-\alpha) \mathbf{e}_{\theta}\right) \\
=U_{0}(\cos \alpha \hat{\mathbf{n}}+\sin \alpha \hat{\mathbf{b}}) .
\end{array}
$$

The calculation proceeds as for (105)-(107) and leads to

$$
\lim _{R \rightarrow \infty} \mathbf{Q}_{i}^{(1)}=-\frac{U_{0} \beta}{2 N_{k}}(\cos \alpha \hat{\mathbf{b}}-\sin \alpha \hat{\mathbf{n}})+\mathbf{Q}_{i, s}^{(1)}(s) \mathbf{e}_{\mathbf{s}}
$$

Matching this with the solution in the intermediate region we find that

$$
\mathbf{v}^{(0)}=-\frac{2 P N_{k}}{\beta}(\cos \alpha \hat{\mathbf{n}}+\sin \alpha \hat{\mathbf{b}})
$$

where

$$
\mathbf{Q}_{\text {int }}^{(0)}=P(\cos \alpha \hat{\mathbf{b}}-\sin \alpha \hat{\mathbf{n}})+\mathbf{Q}_{\mathrm{int}, s}^{(0)}(s) \mathbf{e}_{\mathbf{s}}
$$


Hence

$$
\mathbf{v}^{(0)}=-\frac{2 N_{k}}{\beta} \mathbf{Q}_{\text {int }}^{(0)} \wedge \hat{\mathbf{t}}=\frac{2 N_{k}}{\beta} \mathbf{J}_{o}^{(0)} \wedge \hat{\mathbf{t}}
$$

where $\mathbf{J}_{o}^{(0)}=-\mathbf{Q}_{o}^{(0)}=$ curl $\mathbf{H}_{o}^{(0)}$ is the leading-order electric current in the outer region. Thus we see that the motion of the vortex is dominated by the mean-field behaviour. Equation (4) closes the model (144)-(145) in the outer region.

Remark. We have considered the cases $\delta \sim 1$ and $\delta^{2} \ll 1 /|\log \epsilon|$. Vortex velocities are dominated by the self-induced motion in the first case and by a mean-field motion in the second. Clearly we could also consider the case $\delta^{2} \sim 1 /|\log \epsilon|$, in which case the self-induced motion and the mean-field motion are of the same order. We would find that the law of motion, as expected, is simply the sum of self-induced and meanfield motions. However, in formulating the outer, mean-field model of vortex motion (144)-(145) we needed to assume that neighbouring vortices point in roughly the same direction and move with the same speed. This will clearly not be the case while the vortex velocity depends on its curvature. Thus, although the case $\delta^{2} \sim 1 /|\log \epsilon|$ is a canonical scaling which bridges the gap between our examples, the outer model is hard to formulate for this scaling of the parameters.

5. Conclusion. We have performed a formal asymptotic expansion of the solution of the Ginzburg-Landau equations for a system of curvilinear vortices in a type II superconductor as the core size of the vortices tends to zero. In this limit the vortices behave as line singularities in the equation for the magnetic field.

This simplified model is then closed by considering a local analysis of each vortex core which results in a law of motion for the singularities. The leading term in this law of motion is a local self-induced term proportional to the curvature of the vortex line and in the direction of the principal normal.

In the corresponding law of motion for vortices in an inviscid fluid the leadingorder term is also a local self-induced motion proportional to the curvature but is directed in this case along the binormal. This difference of behaviour may be illustrated by the following simple example.

Consider the following helical line singularity:

$$
\mathbf{q}(s, t)=(a(t) \cos k(t) s, a(t) \sin k(t) s, c(t) s+d(t)) .
$$

Then

$$
\begin{aligned}
\frac{\partial \mathbf{q}}{\partial t} & =(\dot{a} \cos k s-a \dot{k} \sin k s, \dot{a} \sin k s+a \dot{k} \cos k s, \dot{c} s+\dot{d}) \\
\hat{\mathbf{t}} & =\frac{1}{\sqrt{c^{2}+a^{2} k^{2}}}(-a k \sin k s, a k \cos k s, c) \\
\hat{\mathbf{n}} & =(-\cos k s,-\sin k s, 0) \\
\hat{\mathbf{b}} & =\frac{1}{\sqrt{c^{2}+a^{2} k^{2}}}(c \sin k s,-c \cos k s, a k) \\
C & =\frac{a k^{2}}{c^{2}+a^{2} k^{2}}
\end{aligned}
$$


where a dot represents $d / d t$. For a fluid vortex we have $\mathbf{v}=C \mathbf{b}$, giving

$$
\begin{aligned}
& a=\text { constant } \\
& c=\text { constant } \\
& \dot{k}=\frac{-c k^{2}}{\left(c^{2}+a^{2} k^{2}\right)^{3 / 2}}, \\
& \dot{d}=\frac{a^{2} k^{3}}{\left(c^{2}+a^{2} k^{2}\right)^{3 / 2}} .
\end{aligned}
$$

Hence the amplitude and pitch of the helix remain constant, but the vortex precesses and translates. For the particular case of a circle $(c=0)$ we have $k=$ constant, $d=t / a$, and the vortex translates uniformly. We note that a helix is the only known exact solution of the equations of motion of an isolated fluid vortex [2].

For a superconducting vortex, we have $\mathbf{v}=C \mathbf{n}$, giving

$$
\begin{aligned}
d & =\text { constant } \\
c & =\text { constant } \\
k & =\text { constant } \\
\dot{a} & =\frac{-a k^{2}}{c^{2}+a^{2} k^{2}}
\end{aligned}
$$

Therefore

$$
\frac{c^{2}}{k^{2}} \log a+\frac{a^{2}}{2}=-t+\text { constant. }
$$

Hence the vortex does not precess or translate, the pitch of the helix remains constant, and its amplitude tends to zero in infinite time. For the particular case of a circle we have $a=\sqrt{2\left(t_{0}-t\right)}$, and the vortex extinguishes itself in finite time.

At the next order the law of motion of a superconducting vortex contains three terms. The first is again a local self-induced term, proportional to curvature and along the principal normal. The second is a nonlocal self-induced term which is due to the current generated by the "arms" of the vortex line. The third is a term due to the current generated by all the other vortices present.

Unlike fluid vortices, for which the corresponding terms give the vortex a velocity equal to the underlying fluid velocity, vortices in a superconductor do not move with the current but in a direction perpendicular to both it and the vortex line. This change in the direction of motion again leads to behaviour in a system of superconducting vortices very different from that in the corresponding system of fluid vortices. For example, a pair of parallel rectilinear vortices in a superconductor will move along the line of centres (like vortices repel, opposite attract), whereas the corresponding motion of fluid vortices would be perpendicular to the line of centres.

Finally, we considered a situation in which the density of vortices was such that the self-induced term in the law of motion was replaced by the motion due to the mean-field as the leading term. Such a situation is important when we formulate a mean-field model of superconducting vortices, in which the individual vortices are replaced by a vortex density, as in [5]. 
Acknowledgement. The authors would like to thank Dr J. R. Ockendon for his helpful comments during the preparation of this manuscript.

\section{REFERENCES}

[1] J. Bardeen, L. N. Cooper, And J. R. Schrieffer, Theory of superconductivity, Phys. Rev., 108 (1957), p. 1175.

[2] G. K. Batchelor, An Introduction to Fluid Dynamics, Cambridge University Press, Cambridge, UK, 1967.

[3] M. S. BERGER AND Y. Y. CHEN, Symmetric vortices for the Ginzburg-Landau equations of superconductivity and the nonlinear desingularisation phenomenon, J. Funct. Anal., 82 (1989), pp. 259-295.

[4] S. J. Chapman, S. D. Howison, And J. R. Ockenden, Macroscopic models for superconductivity. SIAM Rev., 34 (1992), pp. 529-560.

[5] S. J. ChAPMAN, A mean-field model of superconducting vortices in three dimensions, SIAM J. Appl. Math., 55 (1995), pp. 1259-1274.

[6] X. Chen, C. M. ElliotT, AND Q. TANG, Shooting method for vortex solutions of a complexvalued Ginzburg-Landau equation, 1994, preprint.

[7] Q. Du, M. D. Gunzburger, And J. S. Peterson, Analysis and approximation of the GinzburgLandau model of superconductivity, SIAM Rev., 34 (1992), p. 54.

[8] A. T. DoRsEy, Vortex motion and the Hall effect in type-II superconductors: A time-dependent Ginzburg-Landau theory approach, Phys. Rev. B, 46 (1992), p. 8376.

[9] V. L. GinzBurg And L. D. LAndau, On the theory of superconductivity, J. Experiment. Theoret. Phys., 20 (1950), p. 1064.

[10] L. P. Gor'Kov AND G. M. EliAshBURG, Generalisation of the Ginzburg-Landau equations for non-stationary problems in the case of alloys with paramagnetic impurities, Sov. Phys. J.E.T.P., 27 (1968), p. 328.

[11] L. P. GOR'KOV AND N. B. KOPNIN, Viscous vortex flow in superconductors with paramagnetic impurities, Sov. Phys. J.E.T.P., 33 (1971), p. 1251.

[12] L. Peres And J. Rubinstein, Vortex dynamics in U(1) Ginzburg-Landau models. Phys. D, 64 (1993), pp. 299-309.

[13] P. G. Saffman, Vortex Dynamics, Cambridge University Press, Cambridge, UK, 1992.

[14] M. VAN DyKe, Perturbation Methods in Fluid Dynamics, Parabolic Press, Stanford, CA, 1975. 